\title{
The effectiveness of e-\& mHealth interventions to promote physical activity and healthy diets in developing countries: A systematic review
}

Andre Matthias Müller ${ }^{1 *}$, Stephanie Alley ${ }^{2}$, Stephanie Schoeppe ${ }^{2}$ and Corneel Vandelanotte ${ }^{2}$

\begin{abstract}
Background: Promoting physical activity and healthy eating is important to combat the unprecedented rise in NCDs in many developing countries. Using modern information-and communication technologies to deliver physical activity and diet interventions is particularly promising considering the increased proliferation of such technologies in many developing countries. The objective of this systematic review is to investigate the effectiveness of e-\& mHealth interventions to promote physical activity and healthy diets in developing countries.

Methods: Major databases and grey literature sources were searched to retrieve studies that quantitatively examined the effectiveness of e-\& mHealth interventions on physical activity and diet outcomes in developing countries. Additional studies were retrieved through citation alerts and scientific social media allowing study inclusion until August 2016. The CONSORT checklist was used to assess the risk of bias of the included studies.

Results: A total of 15 studies conducted in 13 developing countries in Europe, Africa, Latin-and South America and Asia were included in the review. The majority of studies enrolled adults who were healthy or at risk of diabetes or hypertension. The average intervention length was 6.4 months, and text messages and the Internet were the most frequently used intervention delivery channels. Risk of bias across the studies was moderate ( $55.7 \%$ of the criteria fulfilled). Eleven studies reported significant positive effects of an e-\& mHealth intervention on physical activity and/or diet behaviour. Respectively, $50 \%$ and $70 \%$ of the interventions were effective in promoting physical activity and healthy diets.

Conclusions: The majority of studies demonstrated that e-\& mHealth interventions were effective in promoting physical activity and healthy diets in developing countries. Future interventions should use more rigorous study designs, investigate the cost-effectiveness and reach of interventions, and focus on emerging technologies, such as smart phone apps and wearable activity trackers.
\end{abstract}

Trial registration: The review protocol can be retrieved from the PROSPERO database (Registration ID: CRD42015029240).

Keywords: Web-based, Mobile phone, ICT, Technology, Developing countries, Dietary transition, NCDs, Exercise, Healthy eating

\footnotetext{
* Correspondence: andrematthiasmueller@gmail.com

${ }^{1}$ Centre for Community and Clinical Applications of Health Psychology;

Faculty of Social, Human and Mathematical Sciences, University of

Southampton, Southampton SO17 1BJ, UK

Full list of author information is available at the end of the article
} 


\section{Background}

In 2012, about 38 million global deaths were attributed to non-communicable diseases (NCDs) such as diabetes, cardiovascular diseases and cancer, and it is expected that NCD death rates will increase further, reaching 52 million by 2030. The NCD burden is particularly high in developing countries with $82 \%$ of global NCD-related deaths occurring in low and middle income countries $[1,2]$.

Major NCD prevention strategies include the reduction of behavioural risk factors, especially physical inactivity and unhealthy diets [1,3-6]. There is extensive evidence on the preventive effects of regular physical activity and healthy eating on the risk of developing a NCD [7, 8]. For example, a $25 \%$ reduction in physical inactivity is estimated to prevent about 1.3 million NCD-related deaths annually [9] while a healthy diet and increased physical activity can prevent a significant proportion of the 18 million deaths caused by high blood pressure, high body mass index, high fasting blood glucose and high total cholesterol [10].

In developing countries, rapid globalization is contributing to a change in people's diets where local low calorie and high fibre foods are replaced by readily available, cheap and processed foods high in fat, salt and sugar [8, 11]. For example, in developing countries in Asia, the consumption of processed foods increased by more than $5 \%$ between 1999 and 2012. In contrast, the consumption of processed foods in developed countries increased only by $0.2 \%$ [12]. Additionally, rapid technological development decreases the necessity of physical labour and active transport which in turn contributes to decreasing levels of physical activity $[8,13,14]$. Decreasing physical activity levels were observed in most Asian [15, 16], Latin-and South American [17-19] and some African countries [13, 20] as urbanization increased.

One promising way to promote physical activity and healthy diets in developing countries is to implement electronic and mobile health (e-\& mHealth) interventions. These interventions are primarily delivered via modern information and communication technologies (ICT) such as the Internet, mobile phones, and other wireless devices [21]. The proliferation of such ICTs is very high in developing countries. For example, in 2015, $90 \%$ of people living in developing countries owned a mobile phone and two thirds of the global Internet users were based in developing countries [22, 23]. Therefore, it is feasible, and potentially cost-effective, to reach large numbers of people using ICTs in developing countries.

Currently, the evidence on physical activity and behavioural diet e-\& mHealth interventions is largely drawn from reviews that did not include studies conducted in developing countries [21, 24, 25]. For example, a recent review on mHealth for the prevention of cardiovascular diseases, only retrieved studies conducted in developed countries [24]. The same applies to reviews focusing on Internet [25, 26], social media [27], smart phone [28, 29], and mobile phone text messaging $[30,31]$ interventions to promote physical activity and healthy diets. Furthermore, a recent review focussing on mHealth interventions in patients with an NCD from developing countries identified only two studies that measured physical activity and none examined dietary behaviours [32]. A systematic review of the research literature on physical activity and diet e-\& mHealth interventions conducted in the developing world is currently lacking.

To address this gap, the objective of this systematic review was to investigate the effectiveness of e-\& mHealth interventions to promote physical activity and healthy diets in developing countries.

\section{Methods}

This review was conducted and is reported according to the PRISMA guidelines [33] and the protocol can be retrieved from the PROSPERO database (Registration ID: CRD42015029240).

\section{Study eligibility criteria}

Studies were included if they a) quantitatively examined the effect of an e-\& mHealth intervention on physical activity and/or diet outcomes; b) were conducted as a quasiexperimental trial, cross-over trial, controlled trial (CT), or randomized controlled trial (RCT); c) included participants from a developing country; and d) were published in English. Only studies in which the e-\& mHealth component was the main or a major intervention delivery mode were included. E-\& mHealth was defined as the use of ICT to promote physical activity and/or healthy diets. Interventions that were delivered via the Internet (webpages, social media, and email), mobile phone text messages or mobile phone calls, smartphone technology ('apps') and other wireless devices (e.g. wearable activity trackers, tablets) were included [21]. The current World Bank classification (July 2015) was used to determine developing country status (low income, lower-middle income, and upper-middle income) [34]. Studies were still included when they were simultaneously conducted in developing and developed countries [35-37]. Every search record was assessed against the inclusion criteria by one of the reviewers (AMM, SA, $\mathrm{SS}$ ). In cases where study inclusion was unclear a decision was made via discussion including all reviewers.

The primary outcomes of this review were objectively or subjectively measured physical activity and/or dietary behaviour. This could be changes in physical activity levels, time spent doing physical activity, adherence to physical activity recommendations, energy expenditure, step counts, exercise/sport participation, active transport, sedentary time, accelerometer counts; food frequency, diet quality (as defined in the respective studies), fruit and vegetable intake, 
consumption of sweetened beverages and foods high in sugar, salt or saturated fat (or ultraprocessed foods), dairy product consumption, consumption of fat and dietary fibre, meal size, or caloric intake. Indirect calorimetry, body composition, BMI, body weight, waist circumference, waist-hip ratio, body fat, and lean body mass were reported if available, but only as secondary outcomes.

\section{Information sources and search}

A systematic search was performed in the following databases: Cochrane Central Register of Controlled Trials (CENTRAL) and the Health Technology Assessment Database (HTA; Cochrane Library), EBSCOHOST (including SPORTDiscuss, CINAHL, MEDLINE, PsycARTICLES, Psychology and Behavioral Science Collection), SCOPUS, Web of Science Core Collection and the World Health Organization Global Health Library. For each database, search terms were combined with the appropriate Boolean Operators: technology or email or internet etc. AND physical activity or exercise or walking etc. or healthy eating or nutrition or sugar intake etc. AND developing country or low-income country etc. or Afghanistan or Albania etc. The search period covered the date range 2000 to 31st October 2015 where available in the database. The data base search (Additional file 1 Cochrane Library) was piloted by the corresponding author and reviewed by all co-authors.

Additionally, articles were hand-searched in the grey literature and reference lists of relevant papers were reviewed. Moreover, the Johns Hopkins Global mHealth Initiative was contacted and a request for relevant studies was posted on ResearchGate (social media network for scientists) and created article alerts to derive 2016 articles outside the systematic search to be as current as possible (last inclusion was made in August 2016).

\section{Data extraction}

A data extraction form was developed based on previous reviews on physical activity and diet interventions $[29,30]$. It was piloted on four studies by the corresponding author and refinements were made based on the feedback of the co-authors. Data on study setting/location and participants, e-\& mHealth intervention characteristics and intervention effectiveness were retrieved. One reviewer extracted the relevant study information and a second reviewer assessed the data for accuracy and completeness. Disagreement between the reviewers was resolved through discussion and consensus with a third reviewer.

The risk of bias assessment was conducted independently by two reviewers using the CONSORT checklist (AMM, SA) [38] which has been used in previous e-\& mHealth reviews [27]. This checklist consists of 25 criteria. If a study fulfilled a criterion it received one point. Studies received half a point if it fulfilled one of two points making up a criterion. A higher overall score indicated lower methodological bias. The obtained risk of bias score of each study was divided by 25 (highest attainable score) and multiplied by 100 to obtain the percentage of fulfilled criteria. Disagreement between the reviewers was resolved through discussion and consensus with a third reviewer (SS). Studies were then grouped into low ( $>66.7 \%$ fulfilled criteria), moderate (50-66.7 \% fulfilled criteria) and high risk of bias ( $<50 \%$ fulfilled criteria) $[25,39]$.

If available, changes in physical activity and diet between baseline and intervention completion, and between baseline and final follow-up (period following an intervention) were presented. If possible, effect sizes were reported or calculated (e.g., Cohen's $d$, mean difference of change between groups), and confidence intervals and significance levels were presented. Where possible the between group effects were presented. The significance level was set to $p \leq .05$.

As few studies provided data needed to calculate effects sizes and due to the great variability in study designs, interventions, and outcome measures conducting a metaanalysis was not possible.

\section{Results \\ Study selection}

A total of 5961 publications including 2231 duplicates were identified through the date base search. After screening the titles and abstracts of 3858 publications, the fulltext of 31 publications was assessed for study eligibility. Of these, 10 publications were included in the review. Another six publications were identified through citation alerts and reference list checks. Finally, two publications reported on the same study [40,41]. As such, a total of 16 publications describing 15 distinct studies were included in the current systematic review (Fig. 1).

\section{Study characteristics}

Table 1 provides an overview of the study characteristics. The majority were RCTs $(n=8)$ with two $(n=7)[36,40$, 42-46] or three group study designs $(n=1)$ [35]. The remaining seven studies were CTs $(n=3)$ [47-49] or quasi-experimental trials $(n=4)$ [37, 50-52]. The e-\& mHealth interventions lasted between 1 and 24 months (median $=4$ months, mean $=6.4$ months). In eleven studies physical activity and/or diet outcomes were assessed only at baseline and immediately after the e-\& mHealth intervention [35-37, 40, 42, 45, 47-49, 51, 52]. Three studies also conducted follow-up assessments after intervention conclusion (3-month follow-up for all 3 studies) $[44,46,50]$ and in one study, physical activity and diet outcomes were assessed at baseline, during the intervention and at intervention completion [43].

One study was conducted in Europe (Turkey) [52], one in Africa (South Africa) [50], three in Latin-or South America (Mexico, Brazil, Peru, and Guatemala) [35, 36, 48], 


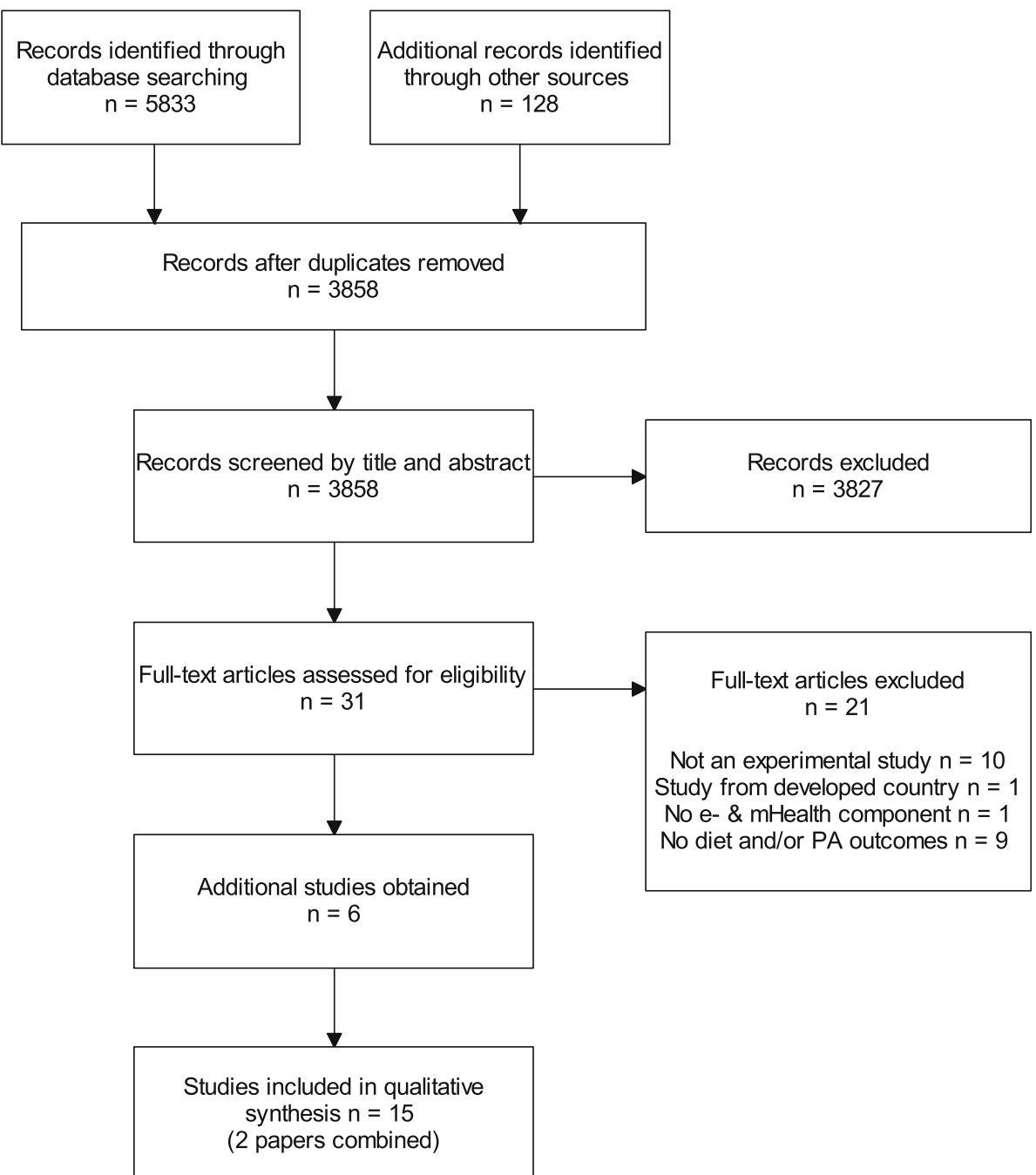

Fig. 1 Flowchart for the selection of studies on e-\& mHealth interventions to promote physical activity and healthy diets in developing countries

nine in Asia (India, Iran, China, Philippines, Thailand, Pakistan, and Malaysia) [40, 42-47, 49, 51] and one across a large number of countries [37]. Rubinstein et al. [36] conducted their study in Peru, Guatemala and Argentina, Lana et al. [35] in Spain and Mexico, and Ganesan et al., [37] included participants from 64 countries with $92 \%$ from a developing country (mainly India). The number of participants ranged from 22 [50] to 69219 [37] with four trials enrolling less than 100 participants [46, 47, 50, 52]. In total 75930 people participated in all included studies. Fourteen studies enrolled adults over 18 years of age (range $=18$ to 74 years), and one study recruited children and adolescents [35]. Study participants were healthy or at risk of developing diabetes or hypertension ( $n=10$ studies) $[35-37,40,44,46,48,49,51,52]$ or diabetic ( $n=5$ studies) $[42,43,45,47,50]$. Three studies enrolled only women $[44,50,52]$ and one enrolled only men [40].
The e- \& mHealth interventions were delivered via mobile phone text messages ( $n=7$ studies) [36, 40, 42, 43, 46, 49, 50], the Internet including websites and email ( $n=6$ studies) $[35,37,44,48,51,52]$, a website plus mobile phone text messages $(n=1)$ [35], mobile phone calls $(n=2)$ [45, 47], or mobile phone calls plus text messages $(n=1)$ [47]. In eight studies, limited face-to-face contact or printed media were also part of the e-\& mHealth intervention [36, 40, 42, 43, 45, $46,50,51]$. All studies provided information on the intensity of the e-\& mHealth intervention. For example, in studies that used mobile phone text-messages as intervention delivery channel text-messaging frequency ranged from twice weekly to daily (mean $=4.5$ text messages per week). Only five studies were theoretically framed [35, 36, 40, 44, 48] with the Transtheoretical Model of Behavior Change most frequently applied ( $n=3$ studies) [35, 36, 40]. However, in other interventions behaviour change techniques (BCTs) 
Table 1 Characteristics of behavioural e-\& mHealth intervention studies included in the review

\begin{tabular}{|c|c|c|c|c|c|}
\hline Author Year Country & $\begin{array}{l}\text { Study design Duration } \\
\text { Sample (sample size, } \\
\text { mean age/range, sex) }\end{array}$ & Intervention & $\begin{array}{l}\text { Outcome measures } \\
\text { (PA, SB, diet) }\end{array}$ & $\begin{array}{l}\text { Retention rate } \\
\text { Acceptability } \\
\text { Participation rate }\end{array}$ & Results \\
\hline $\begin{array}{l}\text { Lana et al. } \\
2014 \text { [35] } \\
\text { Mexico } \\
\text { and Spain }\end{array}$ & $\begin{array}{l}\text { Study design } \\
\text { 3-group RCT } \\
\text { Duration } \\
\text { Intervention exposure: } \\
9 \text { months } \\
\text { Measurement points: } \\
\text { baseline and } 9 \text { months } \\
\text { Sample } \\
N=2001 \text { (737 analysed); } \\
12-16 \text { years students; } \\
45.2 \%(\mathrm{M}), 54.8 \%(\mathrm{~F})\end{array}$ & $\begin{array}{l}\text { IG } 1 \text { \& IG 2: Website targeting } \\
\text { cancer risk behaviours (advantages } \\
\text { of healthy/disadvantages of risky } \\
\text { behaviours, skills training to avoid } \\
\text { risk behaviours, expert advice, } \\
\text { videos, forums, documents, web } \\
\text { links, educational games) based } \\
\text { on the Theory of Planned Behavior } \\
\text { and the Transtheoretical Model } \\
\text { IG 2: Additional weekly text } \\
\text { messages encouraging health } \\
\text { behaviours } \\
\text { CG: No intervention }\end{array}$ & $\begin{array}{l}\text { Behavioural outcomes } \\
\text { Diet (fruit and vegetable } \\
\text { intake, fat intake); PA (doing } \\
\text { PA less than } 360 \text { min/week) } \\
\text { Measures } \\
\text { Online questionnaire } \\
\text { Other relevant outcomes } \\
\text { BMI }\end{array}$ & Retention $36.8 \%$ & $\begin{array}{l}\text { Diet: Sig. within-group increase } \\
\text { in percentage of students } \\
\text { consuming enough fruits in all } \\
\text { groups }(67.0 \% \text { mean decrease, } \\
p<.001) \text {; no sig. within-group } \\
\text { changes for other diet behaviours } \\
\text { PA: No sig. within-groups change } \\
\text { in percentage of students doing } \\
\text { less than } 360 \text { min/week PA } \\
\text { BMl: Sig. within-group changes } \\
\text { in percentage of overweight/obese } \\
\text { students in IG } 2 \text { (19.6\% decrease, } \\
p<.05)\end{array}$ \\
\hline $\begin{array}{l}\text { Rotheram-Borus } \\
\text { et al. } 2012 \text { [50] } \\
\text { South Africa }\end{array}$ & $\begin{array}{l}\text { Study design } \\
\text { 1-group pre-post- } \\
\text { follow-up } \\
\text { Duration } \\
\text { Intervention exposure: } \\
3 \text { months; } \\
\text { Measurement points: } \\
\text { baseline, } 3 \text { and } 6 \text { months } \\
N=22 \text {; } \\
53.0 \text { year } / 21-74 \text { years } \\
\text { diabetic township residents; } \\
100 \% \text { (F) }\end{array}$ & $\begin{array}{l}\text { 3-component PA and diet } \\
\text { program: Weekly educational } \\
\text { group sessions addressing healthy } \\
\text { lifestyle; daily text messages asking } \\
\text { about adherence to healthy } \\
\text { behaviours; peer support for } \\
\text { lifestyle changes via text } \\
\text { messages or call }\end{array}$ & $\begin{array}{l}\text { Behavioural outcomes } \\
\text { PA (daily step count) } \\
\text { Measurements } \\
\text { Pedometer } \\
\text { Other relevant outcomes } \\
\text { BMI }\end{array}$ & $\begin{array}{l}\text { Retention } \\
100 \% \\
\text { Participation rate } \\
\text { Participants responded } \\
\text { to } 54 \% \text { of text messages } \\
\text { sent by study team; peers } \\
\text { exchanged on average } \\
123 \text { text messages weekly }\end{array}$ & $\begin{array}{l}\text { PA: No sig. change in daily step } \\
\text { counts from baseline to } 3 \text { months } \\
(d=0.03) \text {, and from baseline to } \\
6 \text { months ( } d=0.27) \\
\text { BMl: No sig. changes } \\
(d=-0.07 \text { to } 0.07)\end{array}$ \\
\hline $\begin{array}{l}\text { Ramachandran } \\
\text { et al. 2013; } \\
\text { Ram et al. } \\
2014 \text { [40, 41] } \\
\text { India }\end{array}$ & $\begin{array}{l}\text { Study design } \\
\text { Prospective 2-group RCT } \\
\text { Duration } \\
\text { Mean duration of follow-up } \\
20.2 \text { months (SD 7.0), either } \\
\text { intervention exposure of } 24 \\
\text { months or until participants } \\
\text { developed diabetes } \\
\text { Sample } \\
N=537 \text { ( } 517 \text { analysed); } \\
46.0 \text { year/35-55 years working } \\
\text { men with impaired glucose } \\
\text { tolerance; } 100 \%(M)\end{array}$ & $\begin{array}{l}\text { IG \& CG: Face-to-face education } \\
\text { and motivation about healthy } \\
\text { lifestyle plus written information } \\
\text { about diet and PA (balance food } \\
\text { intake and PA to achieve/maintain } \\
\text { healthy body weight } \\
\text { IG: Additional 2-4 weekly text } \\
\text { messages; messages based on } \\
\text { the Transtheoretical Model and } \\
\text { contained information about } \\
\text { diet and PA, benefits of healthy } \\
\text { diet and PA, strategies for relapse } \\
\text { prevention and motivation to } \\
\text { maintain healthy diet and PA }\end{array}$ & $\begin{array}{l}\text { Behavioural outcomes } \\
\text { Diet (total dietary energy } \\
\text { intake, adherence to dietary } \\
\text { recommendations, portion } \\
\text { size, oil intake, carbohydrate } \\
\text { consumption); PA (PA score } \\
\text { ranging from } 7 \text { to } 70 \text {, } \\
\text { adherence to PA recommendations) } \\
\text { Measurements } \\
\text { Diet ( } 24 \text { h recall), PA } \\
\text { (own questionnaire) } \\
\text { Other relevant outcomes } \\
\text { BMI, waist circumference }\end{array}$ & $\begin{array}{l}\text { Retention } \\
96 \% \\
\text { Acceptability } \\
\text { Test messages were } \\
\text { welcomed, } 3 \% \text { were } \\
\text { disturbed by text } \\
\text { messages at least once }\end{array}$ & 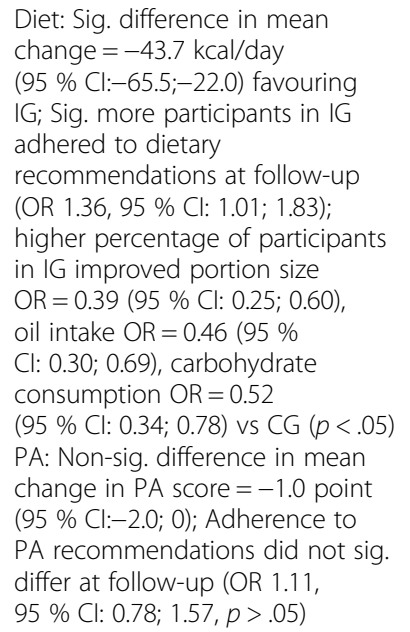 \\
\hline
\end{tabular}


Table 1 Characteristics of behavioural e-\& mHealth intervention studies included in the review (Continued)

\begin{tabular}{|c|c|c|c|c|c|}
\hline $\begin{array}{l}\text { Shetty et al. } \\
2011 \text { [42] } \\
\text { India }\end{array}$ & $\begin{array}{l}\text { Study design } \\
\text { 2-group RCT } \\
\text { Duration } \\
\text { Intervention exposure: } \\
12 \text { months } \\
\text { Measurement points: } \\
\text { baseline and } 12 \text { months } \\
\text { Sample } \\
N=215 \text { ( } 144 \text { analyzed); } \\
50.3 \text { years/type } 2 \text { diabetic } \\
\text { patients; Both sexes } \\
\text { (no further information) }\end{array}$ & $\begin{array}{l}\text { IG \& CG: During initial and } \\
\text { follow-up visits education } \\
\text { program with individual } \\
\text { advice on nutrition and PA } \\
\text { IG: Additional 2-4 weekly text } \\
\text { messages; reminders/instructions } \\
\text { to follow regimen of healthy } \\
\text { diet and PA; messages on } \\
\text { healthy habits }\end{array}$ & $\begin{array}{l}\text { Behavioural outcomes } \\
\text { Diet (scores for } \\
\text { components of healthy } \\
\text { diet and frequency of } \\
\text { adherence to it); PA } \\
\text { (scores for occupation } \\
\text { and leisure time PA) } \\
\text { Measurements } \\
\text { Questionnaire } \\
\text { Other relevant outcomes } \\
\text { BMl }\end{array}$ & $\begin{array}{l}\text { Retention } \\
67 \% \\
\text { Acceptability } \\
\text { Text messages } \\
\text { highly acceptable } \\
\text { reminder tool }\end{array}$ & $\begin{array}{l}\text { Diet: No sig. changes in percentage } \\
\text { of participants adhering to diet } \\
\text { regiment (IG: from } 60.3 \text { to } 58.4 \% \text {; } \\
\text { CG: from } 54.5 \text { to } 52 \% \text { ) } \\
\text { PA: No sig. changes in percentage } \\
\text { of participants complying } \\
\text { with PA advice (IG: } 47 \text { to } 56 \% \text {; } \\
\text { CG: } 47 \text { to } 52 \% \text { ) } \\
\text { BMI: No sig. changes }\end{array}$ \\
\hline $\begin{array}{l}\text { Zolfaghari } \\
\text { et al. } 2012 \\
\text { [47] Iran }\end{array}$ & $\begin{array}{l}\text { Study design } \\
\text { 2-group CT } \\
\text { Duration } \\
\text { Intervention exposure: } \\
3 \text { months } \\
\text { Measurement points: } \\
\text { baseline and } 3 \text { months } \\
\text { Sample } \\
N=80 \text { (77 analysed); } \\
18-65 \text { years diabetes } \\
\text { patients; } 53 \% \text { (F) } \\
47 \%(M)\end{array}$ & $\begin{array}{l}\text { IG1: Phone counselling about } \\
\text { diabetes management including } \\
\text { health behaviour twice weekly } \\
\text { for } 1 \text { st month and weekly for } \\
\text { months } 2 \text { and } 3 \text {. } \\
\text { IG2: } 6 \text { weekly text messages } \\
\text { on diabetes management } \\
\text { including behavioural health. }\end{array}$ & $\begin{array}{l}\text { Behavioural outcomes } \\
\text { Adherence to diet and } \\
\text { PA recommendations as } \\
\text { a score } \\
\text { Measurements Questionnaire }\end{array}$ & $\begin{array}{l}\text { Retention } \\
96.3 \%\end{array}$ & $\begin{array}{l}\text { Diet Adherence: Sig. within-group } \\
\text { increase in IG1 }(18.24 \pm 2.46, p<.001) \\
\text { and IG2 (16.50 } \pm 1.98, p<.001) \text { but } \\
\text { no sig. between-group changes } \\
\text { ( } d=-0.78, p=.44) \\
\text { PA Adherence: Sig. within-group } \\
\text { increase in IG1 (35.66 } \pm 0.68, p<.001) \\
\text { and IG2 ( } 40.02 \pm 1.43, p<.001) \text { but } \\
\text { no sig. between-group changes } \\
\text { ( } d=4.13, p=.33)\end{array}$ \\
\hline $\begin{array}{l}\text { Chen et al. } \\
2014 \text { [51] } \\
\text { China }\end{array}$ & $\begin{array}{l}\text { Study design } \\
\text { 1-group pre-post } \\
\text { Duration } \\
\text { Intervention exposure: } \\
1 \text { to } 6 \text { months } \\
\text { Measurement points: } \\
\text { baseline and } 1 \text { to } 6 \text { months } \\
\text { Sample } \\
N=253 ; 40+\text { yrs pre } \\
\text { diabetic patients in } \\
\text { rural area; } 68 \% \text { (F) } 32 \% \text { (M) }\end{array}$ & $\begin{array}{l}\text { Computer tailored web-based } \\
\text { intervention for diabetes } \\
\text { prevention. Delivered each } \\
\text { time a patient presents at } \\
\text { medical clinic to see general } \\
\text { practitioner. Includes education, } \\
\text { diabetes risk scoring and } \\
\text { tailored feedback on changes } \\
\text { on lifestyle behaviours } \\
\text { (diet and PA) and barriers. } \\
\text { Prompts general practitioner. }\end{array}$ & $\begin{array}{l}\text { Behavioural outcomes } \\
\text { PA (one question at follow } \\
\text { up to determine increased } \\
\text { leisure time exercise) } \\
\text { Diet (two questions at follow } \\
\text { up to determine reduced } \\
\text { calorie intake and increased } \\
\text { fruit and vegetable intake) } \\
\text { Measurements } \\
\text { Interview } \\
\text { Other relevant outcomes } \\
\text { Body weight, BMl }\end{array}$ & $\begin{array}{l}\text { Retention } \\
91 \% \\
\text { Acceptability } \\
8.76-9.20 \text { out of } 10 .\end{array}$ & $\begin{array}{l}\text { PA: Sig. change in number of } \\
\text { participants who increased leisure } \\
\text { time exercise from } 16(6.3 \%) \\
\text { to } 49(21.2 \%, p<.001) \\
\text { Diet: Sig. increase in number } \\
\text { participants who reduced caloric } \\
\text { intake from } 4(1.6 \%) \text { to } 165 \\
(71.4 \%, p<.001) \text {; Sig. increase } \\
\text { in number of participants who } \\
\text { increased fruit-and vegetable } \\
\text { intake from } 43(17 \%) \text { to } 205 \\
(88.7 \%, p<.001) \\
\text { BMl: Sig. reduction from } 24.8 \mathrm{~kg} / \mathrm{m}^{2} \\
( \pm 3.21) \text { to } 23.4 \mathrm{~kg} / \mathrm{m}^{2}( \pm 2.95) \\
(d=0.49, p<.001)\end{array}$ \\
\hline
\end{tabular}

BMl: Difference in mean

change $=-0.05 \mathrm{~kg} / \mathrm{m}^{2}$

(95\% Cl: $-0.46 ; 0.37$ )

Waist circumference: Difference

in mean change $=0.04 \mathrm{~cm}$

(95\% Cl: $-0.56 ; 0.64)$

The mean lifestyle score was

higher in the IG than the CG

$(2.59 \pm 1.13$ vs. $2.28 \pm 1.17 ; p=.002)$

components of health

et and frequency of

$N=215$ (144 analyzed):

IGI: Phone counselling about

ntervention exposure:

3 months

$=80$ (77 analysed);

patients; $53 \%$ (F)

Acceptability

Intervention exposure:

1 to 6 months

Diet (two questions at follow

baseline and 1 to 6 months

ther relevant outcomes 
Table 1 Characteristics of behavioural e-\& mHealth intervention studies included in the review (Continued)

\begin{tabular}{|c|c|c|}
\hline $\begin{array}{l}\text { Tamban et al. } \\
2013 \text { [43] } \\
\text { Philippines }\end{array}$ & $\begin{array}{l}\text { Study design } \\
\text { 2-group RCT } \\
\text { Duration } \\
\text { Intervention exposure: } \\
6 \text { months } \\
\text { Measurement points: } \\
\text { baseline, } 3 \text { months } \\
\text { and } 6 \text { months } \\
\text { Sample } \\
N=125 \text { (104 analysed); } \\
19-50 \text { year diabetes } \\
\text { patients; } 48 \% \text { (F) } \\
52 \%(M) .\end{array}$ & $\begin{array}{l}\text { IG \& CG: Lecture from } \\
\text { diabetes educator and } \\
\text { usual appointments with } \\
\text { diabetes educator and } \\
\text { endocrinologist. } \\
\text { IG: Additional text messages } \\
3 \text { times weekly for } 6 \text { months } \\
\text { on healthy diet, exercise and } \\
\text { consequences of negative } \\
\text { health behaviours. }\end{array}$ \\
\hline $\begin{array}{l}\text { Nurgul et al. } \\
2015 \text { [52] } \\
\text { Turkey }\end{array}$ & $\begin{array}{l}\text { Study design } \\
\text { 1-group pre-post } \\
\text { Duration } \\
\text { Intervention exposure: } \\
3 \text { months } \\
\text { Measurement points: } \\
\text { baseline and } 3 \text { months } \\
\text { Sample } \\
N=44 \text { (30 analysed); } \\
\text { 18-55 years university } \\
\text { employees; } 100 \% \text { (F) }\end{array}$ & $\begin{array}{l}\text { Web-based health intervention: } \\
\text { Modules delivered every } 3 \text { weeks. } \\
1 \text { module on nutrition, } 1 \text { on diet } \\
\text { and } 1 \text { on smoking and stress. } \\
\text { Modules consist of an audio- } \\
\text { visual lecture. }\end{array}$ \\
\hline $\begin{array}{l}\text { Bombem et al. } \\
2013 \\
\text { [48] Brazil }\end{array}$ & $\begin{array}{l}\text { Study design } \\
\text { 2-group CT } \\
\text { Duration } \\
\text { Intervention exposure: } \\
6 \text { months } \\
\text { Measurement points: } \\
\text { baseline and } 6 \text { months } \\
\text { Sample } \\
N=279 \text { (236 analysed); } \\
18-64 \text { years adult } \\
\text { employees; } \\
42.3 \%(M), 57.7 \% \text { (F) }\end{array}$ & $\begin{array}{l}\text { IG: Healthy Weight Program } \\
\text { incl. dietary and PA education } \\
\text { through tailored monthly } \\
\text { email messages, as well as goal } \\
\text { setting, and self-monitoring } \\
\text { of weight. Based on Social } \\
\text { Cognitive Theory. } \\
\text { CG: Wait-list control } \\
\text { Healthy weight program } \\
\text { at the end of 6-months } \\
\text { intervention }\end{array}$ \\
\hline $\begin{array}{l}\text { Sriramatr et al. } \\
2014 \text { [44] } \\
\text { Thailand }\end{array}$ & $\begin{array}{l}\text { Study design } \\
\text { 2-group RCT } \\
\text { Duration } \\
\text { Intervention exposure: } \\
3 \text { months }\end{array}$ & $\begin{array}{l}\text { IG: Website and weekly } \\
\text { emails incl. PA education, } \\
\text { tailored advice, goal setting } \\
\text { and self-monitoring via } \\
\text { pedometer. Based on the } \\
\text { Social Cognitive Theory. }\end{array}$ \\
\hline
\end{tabular}

\author{
Behavioural outcomes \\ PA (adherence to 30 mins \\ of exercise on 5 days weekly) \\ Diet (Number of meals meeting \\ diet recommendations an \\ number of days adhered \\ to 3 proper meals recommendation \\ Measurement \\ Interview \\ Other relevant outcomes \\ BMl, Body weight
}

Behavioural outcomes

PA and Diet (Health

Promotion Lifestyle Profile)

Measurement

Online Questionnaire

Retention

$68.2 \%$

Behavioural outcomes

Diet (food and beverage intake

incl. fruits, vegetables, grains,

dairy, meat, legumes; fat

and sodium intake)

Measures

$24 \mathrm{~h}$ dietary recall, phone interview

Retentio

$85 \%$

Behavioural outcomes

PA (daily step count,

weekly leisure-time PA score)

Measurements

Online Questionnaire

Pedometer
Body weight: Sig. reduction from

$62.1 \mathrm{~kg}( \pm 9.85)$ to $58.3 \mathrm{~kg}( \pm 9.18)$

$(d=3.43, p<.001)$

PA: Sig. between-group increase in minutes of exercise at 6 months

favouring the IG $(p=.02)$; no sig.

between-group changes in mean

number of days meeting PA

recommendations

Diet: Sig. between-group

improvements in adherence

to 3 meals per day recommendation

favouring IG $(p=.02)$;

no sig. between-group changes in

mean number of days meeting diet

recommendations.

BMI: No sig. between-within

group changes

Body weight: No sig. between-

within group changes

PA: Sig. increase from 16.63 points

$( \pm 5.33)$ to 19.20 points $( \pm 5.25)$,

$d=-0.48, p=.004$

Diet: Sig. increase from 20.70

points ( \pm 3.90$)$ to 23.47 points

$(+3.41), d=-0.81, p=.001$

Diet: Sig. decrease in overal

diet quality score in both

groups $(p<.05)$. Sig. more

decrease in diet quality score in CG

compared to IG (adjusted impact:

3.55, 95 \% Cl: 1.52; 5.57). Sig. increase

in grains, but decrease in vegetable

consumption, meat, eggs, sodium

intake, and overall diet quality

score $(p<.05)$.

PA daily step counts: Mean

difference in change from

baseline to 3 months between

groups was 3766 steps favouring

IG. Mean difference in change

from baseline to 6 months 
Table 1 Characteristics of behavioural e-\& mHealth intervention studies included in the review (Continued)

Measurement points:

baseline, 3 and 6 months

Sample

$N=110$

19.0 year/18-24 years

students; $100 \%$ (F)

$\begin{array}{ll}\text { Shahid et al. } & \text { Study design } \\ \text { 2015 [45] } & \text { 2-group RCT } \\ \text { Pakistan } & \text { Duration } \\ & \text { Intervention exposure: } \\ & 4 \text { months } \\ & \text { Measurement points: } \\ & \text { baseline and } 4 \text { months } \\ & \text { Sample } \\ & \text { N=440; } \\ & 49.08 \text { years/18-70 year, } \\ & \text { type-2 diabetes patients; } \\ & 61.4 \% \text { (M), 38.6 \% (F) } \\ & \\ & \\ \text { Müller et al. } & \text { Study design } \\ \text { 2016 [46] } & \text { 2-group RCT } \\ \text { Malaysia } & \text { Duration } \\ & \text { Intervention exposure } \\ & \text { (text messaging): } 3 \text { months } \\ & \text { Measurement points: } \\ & \text { baseline, } 3 \text { and } 6 \text { months } \\ & \text { Sample } \\ & \text { N=43 (39 analysed); } \\ & 63.3 \text { years/55-70 year, } \\ & \text { 26\% (M), 74 \% (F) }\end{array}$

$\begin{array}{ll}\text { Rubinstein et al. } & \text { Study design } \\ 2016[36] & \text { 2-group RCT } \\ \text { Peru, Argentina, } & \text { Duration } \\ \text { Guatemala } & \text { Intervention exposure: } \\ & 12 \text { months } \\ & \text { Measurement points: } \\ & \text { baseline and } 12 \text { months }\end{array}$

$26 \%(\mathrm{M}), 74 \%$ (F)

baseline and 12 months
IG \& CG: Usual care plus

leaflet on diet and a

healthy lifestyle

IG: Additional regular

phone calls to provide

feedback on self-monitored

blood glucose levels over

the past readings of 15 days.

Behavioural outcomes

Diet; PA (if they are

following diet plan

Measures

Not reported

Other relevant outcomes

$\mathrm{BM}$

IG \& CG: Printed exercise booklet with 12 age appropriate exercises IG: Additional 60 encouraging text messages over 3 months content based on effective Behavior Change Techniques

Behavioural outcomes

PA-related energy

expenditure; daily time

spent sitting)

Measures

Exercise diary

International PA

Questionnaire (short)

Other relevant outcomes

BM

IG and CG: Leaflet with information Behavioural outcomes

on adoption of healthy lifestyle PA (weekly MET-minutes)

IG: Additional monthly calls to

Diet (daily intake of

sodium, fat and sugar,

to healthy behaviours (diet and

max. 5 text messages

Measures

per month that were based (every 15 days) mobile

and are physically active)

PA (weekly exercise frequency

using the exercise booklet;

fruits and vegetables) each week

tention

Not reported

between groups was 3360

steps favouring IG

PA leisure time activity score:

Mean difference in change

from baseline to 3 months

between groups was 15.13

points favouring IG. Mean

difference in change from baseline

to 6 months between groups

was 1487 points favouring

Diet: Sig. increase in proportion of

participants following dietary plan

from baseline $(173 \%)$ to 4 month

$(43.6 \%)$ in IG $(p<001)$. Non-sig.

in $C G$

PA: Sig. increase in proportion of

physically active participants from

baseline $(16.4 \%)$ to 4 months

$(44.5 \%)$ in IG $(p<.001)$ Non-sig.

in CG

BMI: Sig. reduction $(p<.001)$ in

IG (.96 + 09) and CG (1.02 \pm .09 ;

favouring $C G$

Retention

$86 \%$

PA (exercise frequency): Sig.

more often exercise in IG $(3.7 \pm 1.3)$

$\begin{array}{ll}\text { Acceptability } & \text { compared to CG }(2.5 \pm 1.85) \text { at } \\ \text { IG participants liked the text } 3 \text { months }(d=0.76, p=.027) \text {; }\end{array}$

$\begin{array}{ll}\text { IG participants liked the text } 3 \text { months }(d=0.76, p=.027) \text {; } \\ \text { messages and those who } & \text { Non-sig. difference at } 6 \text { months }\end{array}$

faced exercise base who

benefited from them.

(3.1 \pm 1.3 vs. $2.3 \pm 1.9, d=0.45$

$p=.18)$

PA (PA related energy

expenditure): No

sig. between-within group changes

PA (daily time spent sitting):

No sig. between-within group changes

BMl: No sig. between-group

changes

Retention: $86.8 \%$

Acceptability. Participar

found call and text

Diet (daily sodium intake): Mean

Participation rate: Only $3 \%$ difference in change between

received all 12 calls, call groups - 0.07 (95\% Cl:-0.25;0.12

duration 20-30 min, $\quad p=.49$ ) 
Table 1 Characteristics of behavioural e-\& mHealth intervention studies included in the review (Continued)

\begin{tabular}{|c|c|c|c|c|c|}
\hline & $\begin{array}{l}\text { Sample } \\
N=637(553 \text { analysed); } \\
43.4 \text { years/30-60 year, } \\
\text { adults with prehypertension } \\
46 \%(M), 54 \%(F)\end{array}$ & $\begin{array}{l}\text { on the Transtheoretical Model } \\
\text { (target on chosen diet/ } \\
\text { PA behaviour). }\end{array}$ & $\begin{array}{l}\text { International PA } \\
\text { Questionnaire (short) } \\
\text { Food Frequency } \\
\text { Questionnaire } \\
\text { Other relevant outcomes } \\
\text { BMl, body weight, } \\
\text { waist circumference }\end{array}$ & $\begin{array}{l}\text { median of } 23 \text { text } \\
\text { messages over } 12 \text { months }\end{array}$ & $\begin{array}{l}\text { Diet (daily fat and sugar intake): } \\
\text { Mean difference in change between } \\
\text { groups }-0.75 \text { ( } 95 \% \text { Cl:-1.30;-0.20, } \\
p=.008) \\
\text { Diet (daily intake of fruits and } \\
\text { vegetables): Mean difference in } \\
\text { change between groups } 0.25 \\
\text { ( } 95 \% \text { Cl:-0.01; } 51, p=.05) \\
\text { BMl: Mean difference in } \\
\text { change between groups }-0.30 \\
(95 \% \mathrm{Cl} \text { : }-0.59 ; 0.06, p=.02) \\
\text { Body weight: Mean difference } \\
\text { in change between groups }-0.66 \\
\text { (95\% Cl: }-1.24 ;-0.07, p=.04) \\
\text { Waist circumference: Mean } \\
\text { difference in change between } \\
\text { groups }-0.64 \text { ( } 95 \% \mathrm{Cl}:-1.62 ; \\
0.35, p=.21)\end{array}$ \\
\hline $\begin{array}{l}\text { Ganesan et al., } \\
2016 \text { [37] } \\
92 \% \text { of participants } \\
\text { from developing } \\
\text { countries } \\
\text { (India, China, } \\
\text { Philippines) }\end{array}$ & $\begin{array}{l}\text { Study design } \\
\text { 1-group pre-post } \\
\text { Duration } \\
\text { Intervention exposure: } \\
2.5 \text { months } \\
\text { Measurement points: } \\
\text { baseline and } 2.5 \text { months } \\
\text { Sample } \\
N=69219 \text { (36652 analysed); } \\
36.0 \text { year ( } \pm 9 \text { years), } \\
\text { adult employees; } \\
76.1 \%(\mathrm{M}), 23.9 \%(\mathrm{~F})\end{array}$ & $\begin{array}{l}\text { 100-day Stepathlon programme: } \\
\text { Participants received pedometer } \\
\text { and entered daily step count } \\
\text { into Stepathlon website or app. } \\
\text { Website to facilitate motivation } \\
\text { and engagement via } \\
\text { self-monitoring, social } \\
\text { networking, quizzes, expert } \\
\text { chats and competition between } \\
\text { employees. Encouraging emails } \\
\text { daily and when milestones } \\
\text { were reached. }\end{array}$ & $\begin{array}{l}\text { Behavioural outcomes } \\
\text { PA (daily step count, weekly } \\
\text { exercise days, daily sitting time) } \\
\text { Measures } \\
\text { Pedometer } \\
\text { Online survey } \\
\text { Other relevant outcomes } \\
\text { Body weight }\end{array}$ & Retention: $53.0 \%$ & $\begin{array}{l}\text { PA (daily step count): } \\
\text { Sig. increase of } 3519 \text { steps } \\
\text { ( } 95 \% \text { Cl: } 3484 ; 3553, p<.001 \text { ) } \\
\text { PA (weekly exercise days): } \\
\text { Sig. increase of } 0.89 \text { days/week } \\
\text { ( } 95 \% \text { Cl: } 0.87 ; 0.92, p<.001 \text { ) } \\
\text { PA (daily sitting time): } \\
\text { Sig. decrease of } 0.74 \text { h/day } \\
\text { steps ( } 95 \% \text { Cl:- } 0.78 ;-0.71, p<.001 \text { ) } \\
\text { Body weight: Sig. reducion } \\
\text { of } 1.45 \mathrm{~kg}(95 \% \text { Cl:- } 1.53 ;- \\
1.38, p<.001)\end{array}$ \\
\hline $\begin{array}{l}\text { Pfammatter } \\
\text { et al., } 2016 \\
\text { [49] India }\end{array}$ & $\begin{array}{l}\text { Study design } \\
\text { 2-group CT } \\
\text { Duration } \\
\text { Intervention exposure: } \\
6 \text { months } \\
\text { Measurement points: } \\
\text { baseline and } 6 \text { months } \\
\text { Sample } \\
N=1925 \text { (1243 analysed); } \\
32.2 \text { years }( \pm 10.6 \text { years); } \\
88.52 \%(M), 11.48 \%(F)\end{array}$ & $\begin{array}{l}\text { IG: } 56 \text { motivational text messages } \\
\text { addressing awareness of } \\
\text { diabetes and diabetes } \\
\text { risk behaviours } \\
\text { CG: No intervention }\end{array}$ & $\begin{array}{l}\text { Behavioural outcomes } \\
\text { PA (current exercise) } \\
\text { Diet (fruit, vegetable } \\
\text { and fat intake) } \\
\text { Measures } \\
\text { Telephone survey }\end{array}$ & Retention: $64.6 \%$ & $\begin{array}{l}\text { PA: No sig. between-group change } \\
\text { in exercise participation }(p>.05) \\
\text { Diet (daily intake of fruit } \\
\text { and vegetables): } \\
\text { Sig. between-group increase } \\
\text { favouring the IG }(p<.001) \\
\text { Diet (fat intake): } \\
\text { Sig. between-group decrease } \\
\text { favouring the IG }(p<.001)\end{array}$ \\
\hline
\end{tabular}


such as goal setting and self-monitoring were used to promote physical activity and healthy diets.

Both physical activity and diet outcomes were assessed in the majority of studies $(n=10)$ [35, 36, 40, 42, 43, 45, 47, $49,51,52]$ while four assessed only physical activity $[37,44$, $46,50]$ and one only diet [48]. Physical activity was assessed in various ways, however most studies used a self-report instrument including questionnaires, interviews and exercise diaries $(n=10)$ [35, 36, 40, 42, 43, 46, 47, 49, 51, 52]. The outcomes also varied greatly across studies: adherence to physical activity guidelines, time spent being active, physical activity-related energy expenditure, physical activity score, and exercise frequency. In one study step-count data were collected with a pedometer [50] while Sriramatr et al. [44] and Ganesan et al. [37] used both pedometer data and a questionnaire. Diet outcomes were only assessed subjectively via questionnaires, 24-h recall sheets and interviews. One study did not report how outcome data was assessed [45]. Secondary outcomes were assessed in ten studies [35-37, 40, 42, 43, 45, 46, 50, 51]. All these studies reported data on BMI and six studies also reported on waist circumference and/or body weight.

\section{Risk of bias within studies}

The detailed CONSORT risk of bias assessment of the individual studies is presented in Additional file 2. On average the studies fulfilled $55.7 \%$ of the assessment criteria (range $=28-88 \%$ ). Hence, overall the studies had a moderate risk of bias with just over half of the studies at high risk $(n=8$ studies) [37, 42, 44, 45, 48, 50-52]. Few studies provided adequate information on intervention harms $(n=2)$ [40, 46], study protocol publication $(n=3)[35,36,51]$, study registration $(n=6)[35-37,40,46,49]$, ancillary analyses $(n=5)[35-37,40,50]$, and randomization as well as blinding $(n=4)[36,40,43,46]$.

\section{Intervention effectiveness}

Of the 15 studies included, four reported no significant positive effects on either physical activity or diet outcomes following an e-\& mHealth intervention [35, 42, 47, 50]. The majority of studies $(n=11)$ reported at least one significant positive effect on physical activity or diet outcomes (Table 2). No clear patterns emerged between those studies that were effective and those that were not. In terms of secondary outcomes BMI was measured in nine studies with two reporting a significant positive effect of an e-\& mHealth intervention despite not focussing on weight loss [36, 51].

\section{Discussion}

Promoting healthy lifestyles is an effective public health strategy to address the NCD rise in developing countries [1]. Given the great proliferation of ICT in developing countries, the use of e-\& mHealth approaches appear to be viable to promote physical activity and healthy diets [21-23]. The results of this systematic review suggest that e-\& mHealth interventions can be effective in improving physical activity and diet quality in developing countries. Overall, this review showed that $50 \%$ of the e-\& mHealth interventions were effective in increasing physical activity, and $70 \%$ of the identified interventions were effective in improving diet quality. This result is consistent with the findings from previous systematic reviews of e-and mHealth interventions conducted in developed countries [21, 24-26, 29, 31, 53-55]. The findings from this review also add to the overall evidence on the effectiveness of e-\& mHealth interventions in developing countries that were conducted to assess other health outcomes (e.g., treatment adherence) [32, 56-61]. As the included studies used multiple intervention components, it was not possible to identify which specific components were associated with interventions effectiveness. However, most Internet-based interventions were effective in improving physical activity and/or diet while the evidence for mobile-phone interventions (text messages and counselling) was mixed.

The overall risk of bias of the included studies was moderate with just over half of the studies having a high risk of bias. Study quality or study bias are also a concern in studies conducted in developed countries. Most previous reviews on the effectiveness of e-\& mHealth interventions to promote physical activity and healthy diets in developed countries reported that the included studies were of low to moderate quality or rather biased $[28,30,55,62,63]$. There was no association between the risk of bias score and the effectiveness of the e-\& mHealth interventions. The four studies with the lowest risk of bias reported mixed results $[35,36,40,46]$ and the same is true for the studies with higher risk of bias.

Importantly, the majority of studies included in this review $(n=10)$ examined an intervention modality that is likely economically viable and has the potential to reach large numbers of people to address the steep increase of NCDs. While more high-quality RCTs are needed to broaden the evidence-base, it is also important to conduct real-life implementation studies, given that the majority of the included studies reported that the e-\& mHealth interventions showed positive outcomes. The study by Ganesan et al. [37] provides an insightful example of real-life implementation of a low-cost e-\& mHealth intervention to increase physical activity. The researchers included almost 70000 participants (92\% from developing countries) into their 100-day Stepathlon programme and found that daily step counts and weekly exercise participation increased greatly. This study was possible because academic researchers teamed up with the private sector and formed a strong collaborative network. To form academicprivate partnerships in order to either conduct or upscale 
Table 2 CONSORT risk of bias assessment and effectiveness of e-\&mHealth interventions on physical activity and diet outcomes

\begin{tabular}{|c|c|c|c|}
\hline Study & Design & $\begin{array}{l}\text { CONSORT score } \\
\text { (percentage of fulfilled criteria) }\end{array}$ & $\begin{array}{l}\text { e-\& mHealth } \\
\text { intervention effectiveness }\end{array}$ \\
\hline \multirow[t]{2}{*}{ Lana et al. 2014 [35] } & \multirow[t]{2}{*}{ 3-group RCT } & \multirow[t]{2}{*}{$18(72 \%)$} & Diet: 0 \\
\hline & & & PA: 0 \\
\hline \multirow[t]{2}{*}{ Rotheram-Borus et al. 2012 [50] } & \multirow[t]{2}{*}{ 1-group pre-post-follow-up } & \multirow[t]{2}{*}{$7.5(30 \%)$} & Diet: Not included \\
\hline & & & PA: 0 \\
\hline \multirow{2}{*}{$\begin{array}{l}\text { Ramachandran et al. 2013; } \\
\text { Ram et al. } 2014 \text { [40, 41] }\end{array}$} & \multirow[t]{2}{*}{ Prospective 2-group RCT } & \multirow[t]{2}{*}{$22(88 \%)$} & Diet: + \\
\hline & & & PA: 0 \\
\hline \multirow[t]{2}{*}{ Shetty et al. 2011 [42] } & \multirow[t]{2}{*}{ 2-group RCT } & \multirow[t]{2}{*}{7 (28 \%) } & Diet: 0 \\
\hline & & & PA: 0 \\
\hline \multirow[t]{2}{*}{ Zolfaghari et al. 2012 [47] } & \multirow[t]{2}{*}{ 2-group CT } & \multirow[t]{2}{*}{$13.5(54 \%)$} & Diet: 0 \\
\hline & & & PA: 0 \\
\hline \multirow[t]{2}{*}{ Chen et al. 2014 [51] } & \multirow[t]{2}{*}{ 1-group pre-post } & \multirow[t]{2}{*}{$10.5(42 \%)$} & Diet: + \\
\hline & & & PA: + \\
\hline \multirow[t]{2}{*}{ Tamban et al. 2013 [43] } & \multirow[t]{2}{*}{ 2-group RCT } & \multirow[t]{2}{*}{$15(60 \%)$} & Diet: + \\
\hline & & & PA: + \\
\hline \multirow[t]{2}{*}{ Nurgul et al. 2015 [52] } & \multirow[t]{2}{*}{ 1-group pre-post } & \multirow[t]{2}{*}{$8.5(34 \%)$} & Diet: + \\
\hline & & & PA: + \\
\hline \multirow[t]{2}{*}{ Bombem et al. 2013 [48] } & \multirow[t]{2}{*}{ 2-group CT } & \multirow[t]{2}{*}{$11.5(46 \%)$} & $\begin{array}{l}\text { Diet: + (less decrease } \\
\text { in diet quality) }\end{array}$ \\
\hline & & & PA: Not included \\
\hline \multirow[t]{2}{*}{ Sriramatr et al. 2014 [44] } & \multirow[t]{2}{*}{ 2-group RCT } & \multirow[t]{2}{*}{$12(48 \%)$} & Diet: Not included \\
\hline & & & $\mathrm{PA}:+$ \\
\hline \multirow[t]{2}{*}{ Shahid et al. 2015 [45] } & \multirow[t]{2}{*}{ 2-group RCT } & $11.5(46 \%)$ & Diet: + \\
\hline & & & $\mathrm{PA}:+$ \\
\hline Müller et al. 2016 [46] & 2-group RCT & $22(88 \%)$ & Diet: Not included \\
\hline & & & PA: + \\
\hline Rubinstein et al. 2016 [36] & 2-group RCT & $22(88 \%)$ & Diet: + \\
\hline & & & PA: 0 \\
\hline Ganesan et al., 2016 [37] & 1-group pre-post & $12(48 \%)$ & Diet: Not included \\
\hline & & & PA: + \\
\hline Pfammatter et al., 2016 [49] & 2-group CT & $16(64 \%)$ & Diet: + \\
\hline & & & PA: 0 \\
\hline
\end{tabular}

$P A$ physical activity, ${ }^{\prime}+{ }^{\prime}=$ significant positive effect of e-\& mHealth intervention, ${ }^{\prime} 0$ ' = no significant effect of e-\& mHealth intervention

e-\& mHealth interventions to increase physical activity and improve diet quality might also be an option for researchers in other developing countries. Benefits of such partnerships include sharing of expertise (academia: behavioural health knowledge, industry: intervention appeal and dissemination expertise) and data sharing which can lead to dynamic intervention development and adaptation [64]. Potential for such partnerships is especially high in countries where there is some preliminary evidence that such interventions are feasible. Feasibility information is available from Malaysia [65-67], Iraq [68], Pakistan [69] and South Africa [70] where the e-\& mHealth interventions were well accepted and study participants found them useful.
A key strength of this review is that this is the first systematic review to investigate the effectiveness of e-\& mHealth interventions to promote physical activity and healthy diets in developing countries. A further strength is that a large number of information sources were systematically searched to identify relevant studies and that the search was updated continuously (until August 2016). Additionally, data of the included studies were extracted in great detail despite the complex nature of some studies and large variations across studies.

A limitation of this review is that only articles published in English were included. Studies conducted in developing countries where English is not the first language might 
have been published in local languages and our search would not have identified them. The possibility of publication bias should also be acknowledged. As with all systematic reviews examining the efficacy of interventions, it is possible that some studies that did not find a beneficial effect of an e-\& mHealth intervention have not been published [71].

Further, studies were only identified in a small number of countries and it is therefore difficult to generalize the review findings across developing countries (most studies were available from Asia). Additionally, most studies were conducted in upper-middle income countries $(n=10)$ and none in a low income country.

Due to the differences in study designs, outcome measures, lengths of studies, and study samples it was difficult to draw clear conclusions in this review. It is therefore important that researchers aim to examine the impact of e-\& mHealth interventions to promote physical activity and healthy diets in a standardized manner. Applying the CONSORT guidelines that specifically highlight the importance of valid controls is strongly encouraged [38]. This will allow for a pooling of results to conduct a meta-analysis, and would reduce the risk of bias in the included studies.

Researchers should be encouraged to assess and report data on intervention acceptability and level of participation (as measured through web and app usage tracking). Only a limited number of studies included in this review presented this information. Knowing how well participants accept and are engaged in e-\& mHealth interventions is essential because acceptability and user engagement are related to intervention effectiveness [72, 73]. Additionally, studies examining the cost-effectiveness and reach of e-\& mHealth interventions in developing countries are currently lacking. These studies are needed to ensure that resource poor countries can impact health behaviours in a large number of people at an affordable cost. From this review, the impact of behavioural e-\& mHealth interventions a) on clinical health outcomes and b) among patients versus nonpatients is unclear and more research is warranted.

Finally, while behavioural e-\& mHealth research in developed countries is well established [74], research in developing countries is only in its infancy. One indication for this is the small number of studies that were identified. In addition, the studies included in this review utilized mainly first generation technologies of the Internet and mobile phones to implement their interventions $(n=14)$. However, with the unprecedented expansion of mobile broadband in developing countries, e-\& mHealth interventions with advanced technologies (e.g. smart phone apps and wearable activity trackers) [23] are likely to emerge [37]. One feasibility study conducted in South Africa already reported that a diet smartphone app for diabetic nephropathy patients is highly acceptable among dietitians [70]. It is important that researchers examine if these advanced technologies can be leveraged to promote physical activity and healthy diets in developing countries.

\section{Conclusions}

In summary, using e-\& mHealth approaches to promote physical activity and healthy diets in developing countries was effective in most studies included in this review. However, the interventions varied greatly in terms of geographic spread, intervention components evaluated, trial methods applied and study quality. Therefore, the findings from the included studies should be interpreted with caution, and more rigorous study designs are recommended for future e-\& mHealth interventions.

\section{Additional files}

Additional file 1: Cochrane Library Complete Search Strategy. (DOCX 18 kb)

Additional file 2: Risk of bias assessment using the CONSORT checklist. (DOCX $32 \mathrm{~kb}$ )

\begin{abstract}
Abbreviations
BMI: Body mass index; CONSORT: Consolidated Standards of Reporting Trials; CT: Controlled trial; e-\& mHealth: Electronic and mobile health;

ICT: Information and communication technology; MET: Metabolic Equivalent of task; NCD: Non-communicable disease; PRISMA: Preferred reporting items for systematic reviews and meta-analyses; RCT: Randomized controlled trial
\end{abstract}

\section{Acknowledgements}

Not applicable.

Funding

$C V$ was supported by a Future Leader Fellowship from the National Heart Foundation of Australia (ID 100427). The funder had no role in any part of the study.

Availability of data and materials

The dataset supporting the conclusions of this article is included within the article (and its Additional files 1 and 2).

\section{Authors' contributions}

AMM developed the concept and design of the study, provided the systematic literature research and drafted the manuscript. AMM, SS and SA extracted the data and rated the risk of bias of individual studies. AMM, SS, SA and CV contributed to the development of the study protocol, interpretation of the data and provided critical revisions to the manuscript. All authors have read and approved the final manuscript.

\section{Competing interests}

The authors declare that they have no competing interests.

\section{Consent for publication}

Not applicable.

Ethics approval and consent to participate Not applicable.

\section{Author details}

${ }^{1}$ Centre for Community and Clinical Applications of Health Psychology; Faculty of Social, Human and Mathematical Sciences, University of Southampton, Southampton SO17 1BJ, UK. ²Physical Activity Research Group, School of Human, Health and Social Sciences, Central Queensland University, Building 77, Bruce Highway, Rockhampton QLD 4702, Australia.

Received: 16 May 2016 Accepted: 5 October 2016

Published online: 10 October 2016 


\section{References}

1. World Health Organization. Global status report on noncommunicable diseases 2014. Geneva: World Health Organization; 2014.

2. Lozano R, Naghavi M, Foreman K, Lim S, Shibuya K, Aboyans V, et al. Global and regional mortality from 235 causes of death for 20 age groups in 1990 and 2010: A systematic analysis for the Global Burden of Disease Study 2010. Lancet. 2012;380(9859):2095-128. http://dx.doi.org/10.1016/501406736(12)61728-0 .

3. Horton R. Non-communicable diseases: 2015 to 2025. Lancet. 2013; 381(9866):509-10. http://dx.doi.org/10.1016/S0140-6736(13)60100-2

4. Balbus JM, Barouki R, Birnbaum LS, Etzel RA, Gluckman PD, Grandjean P, et al. Early-life prevention of non-communicable diseases. Lancet. 2013; 381(9860):3-4. http://dx.doi.org/10.1016/S0140-6736(12)61609-2.

5. Mishra SR, Neupane D, Preen D, Kallestrup P, Perry HB. Mitigation of noncommunicable diseases in developing countries with community health workers. Global Health. 2015;11:43. http://dx.doi.org/10.1186/s12992-015-0129-5.

6. Lachat C, Otchere S, Roberfroid D, Abdulai A, Aguirre Seret FM, Milesevic et al. Diet and physical activity for the prevention of noncommunicable diseases in low-and middle-income countries: a systematic policy review. PLoS Med. 2013;10(6):e1001465. http://dx.doi.org/10.1371/journal.pmed. 1001465

7. Shiroma EJ, Lee I. Physical activity and cardiovascular health: lessons learned from epidemiological studies across age, gender, and race/ethnicity. Circulation. 2010;122(7):743-52. http://dx.doi.org/10.1161/CIRCULATIONAHA. 109.914721

8. Popkin BM. Global nutrition dynamics: the world is shifting rapidly toward a diet linked with noncommunicable diseases. Am J Clin Nutr. 2006;84(2):289-98

9. Lee I, Shiroma EJ, Lobelo F, Puska P, Blair SN, Katzmarzyk PT. Effect of physical inactivity on major non-communicable diseases worldwide: an analysis of burden of disease and life expectancy. Lancet. 2012;380(9838): 219-29. http://dx.doi.org/10.1016/S0140-6736(12)61031-9

10. Lim SS, Vos T, Flaxman AD, Danaei G, Shibuya K, Adair-Rohani H, et al. A comparative risk assessment of burden of disease and injury attributable to 67 risk factors and risk factor clusters in 21 regions, 1990-2010: A systematic analysis for the Global Burden of Disease Study 2010. Lancet. 2012; 380(9859):2224-60. http://dx.doi.org/10.1016/50140-6736(12)61766-8.

11. Steyn NP, McHiza ZJ. Obesity and the nutrition transition in Sub-Saharan Africa. Ann N Y Acad Sci. 2014:1311:88-101. http://dx.doi.org/10.1111/nyas.12433.

12. Baker $P$, Friel $S$. Processed foods and the nutrition transition: evidence from Asia. Obes Rev. 2014;15(7):564-77. http://dx.doi.org/10.1111/obr.12174

13. Hallal PC, Andersen LB, Bull FC, Guthold R, Haskell W, Ekelund U, et al. Global physical activity levels: surveillance progress, pitfalls, and prospects. Lancet. 2012;380(9838):247-57. http://dx.doi.org/10.1016/S0140-6736(12)60646-1

14. Goryakin Y, Lobstein T, James WPT, Suhrcke M. The impact of economic, political and social globalization on overweight and obesity in the 56 low and middle income countries. Soc Sci Med. 2015;133:67-76. http://dx.doi. org/10.1016/j.socscimed.2015.03.030

15. Macniven R, Bauman A, Abouzeid M. A review of population-based prevalence studies of physical activity in adults in the Asia-Pacific region. BMC Public Health. 2012;12(1):41-51. http://dx.doi.org/10.1 186/1471-2458-12-41.

16. Ranasinghe $C D$, Ranasinghe $P$, Jayawardena R, Misra A. Physical activity patterns among South-Asian adults: a systematic review. Int I Behav Nutr Phys Act. 2013;10:1 16. http://dx.doi.org/10.1186/1479-5868-10-116

17. Amorim TC, Azevedo MR, Hallal PC. Physical activity levels according to physical and social environmental factors in a sample of adults living in South Brazil. J Phys Act Health. 2010;7 Suppl 2:S204-12.

18. Masterson Creber RM, Smeeth L, Gilman RH, Miranda JJ. Physical activity and cardiovascular risk factors among rural and urban groups and rural-tourban migrants in Peru: A cross-sectional study. Rev Panam Salud Publica. 2010;28:1. https://www.ncbi.nlm.nih.gov/pmc/articles/PMC2957283/.

19. Bauman A, Bull F, Chey T, Craig CL, Ainsworth BE, Sallis JF, et al. The international prevalence study on physical activity: results from 20 countries. Int J Behav Nutr Phys Act. 2009;6:21. http://dx.doi.org/10.1186/1479-5868-6-21

20. Abubakari AR, Lauder W, Jones MC, Kirk A, Agyemang C, Bhopal RS. Prevalence and time trends in diabetes and physical inactivity among adult West African populations: the epidemic has arrived. Public Health. 2009; 123(9):602-14. http://dx.doi.org/10.1016/j.puhe.2009.07.009

21. Vandelanotte C, Müller AM, Short CE, Hingle M, Nathan N, Williams SL, et al. Past, present and future or e- $\&$ mHealth research to improve physical activity and dietary behaviors. J Nutr Educ Behav. 2016:48(3):219-28. http:// dx.doi.org/10.1016/j.jneb.2015.12.006
22. International Telecommunication Union. The world in 2014: ICT facts and figures. Geneva: International Telecommunication Union; 2014.

23. International Telecommunication Union. ICT facts and figures. Geneva: International Telecommunication Union; 2015.

24. Burke LE, Ma J, Azar KMJ, Bennett GG, Peterson ED, Zheng Y, et al. Current science on consumer use of mobile health for cardiovascular disease prevention: a scientific statement from the American Heart Association. Circulation. 2015;132(12):1157-213. http://dx.doi.org/10.1161/CIR. 0000000000000232

25. Davies CA, Spence JC, Vandelanotte C, Caperchione CM, Mummery WK. Metaanalysis of internet-delivered interventions to increase physical activity levels. Int J Behav Nutr Phys Act. 2012;9:52. http://dx.doi.org/10.1186/1479-5868-9-52.

26. Hou S, Charlery SR, Roberson K. Systematic literature review of Internet interventions across health behaviors. Health Psychol Behav Med. 2014;2(1): 455-81. http://dx.doi.org/10.1080/21642850.2014.895368

27. Maher CA, Lewis LK, Ferrar K, Marshall S, Bourdeaudhuij I, Vandelanotte C. Are health behavior change interventions that use online social networks effective? A systematic review. J Med Internet Res. 2014;16(2):e40. http://dx. doi.org/10.2196/jmir.2952

28. Flores Mateo G, Granado-Font E, Ferré-Grau C, Montaña-Carreras X. Mobile phone apps to promote weight loss and increase physical activity: a systematic review and meta-analysis. J Med Internet Res. 2015;17(11):e253. http://dx.doi.org/10.2196/jmir.4836

29. Bort-Roig J, Gilson ND, Puig-Ribera A, Contreras RS, Trost SG. Measuring and influencing physical activity with smartphone technology: a systematic review. Sports Med. 2014;44(5):671-86. http://dx.doi.org/10.1007/s40279-014-0142-5 .

30. Siopis G, Chey T, Allman-Farinelli M. A systematic review and meta-analysis of interventions for weight management using text messaging. J Hum Nutr Diet. 2015;28(2):1-15. http://dx.doi.org/10.1111/jhn.12207.

31. Weber Buchholz S, Wilbur J, Ingram D, Fogg L. Physical activity text messaging interventions in adults: a systematic review. Worldviews Evid Based Nurs. 2013;10(3):163-73. http://dx.doi.org/10.1111/wvn.12002

32. Stephani V, Opoku D, Quentin W. A systematic review of randomized controlled trials of mHealth interventions against non-communicable diseases in developing countries. BMC Public Health. 2016;16:572. http://dx. doi.org/10.1186/s12889-016-3226-3

33. Liberati A. The PRISMA statement for reporting systematic reviews and meta-analyses of studies that evaluate health care interventions: explanation and elaboration. Ann Intern Med. 2009;151(4):65-94. http://dx.doi.org/10. 7326/0003-4819-151-4-200908180-00136

34. World Bank [Internet]. Country and Lending Groups [cited October 8, 2015]. Available from: http://data.worldbank.org/about/country-and-lending-groups.

35. Lana A, Faya-Ornia G, López ML. Impact of a web-based intervention supplemented with text messages to improve cancer prevention behaviors among adolescents: Results from a randomized controlled trial. Prev Med. 2014:59:54-9. http://dx.doi.org/10.1016/j.ypmed.2013.11.015

36. Rubinstein A, Miranda JJ, Beratarrechea A, Diez-Canseco F, Kanter R, Gutierrez L, et al. Effectiveness of an mHealth intervention to improve the cardiometabolic profile of people with prehypertension in low-resource urban settings in Latin America: A randomised controlled trial. Lancet Diabetes Endocrinol. 2016;4(1): 52-63. http://dx.doi.org/10.1016/S2213-8587(15)00381-2

37. Ganesan AN, Louise J, Horsfall M, Bilsborough SA, Hendriks J, McGavigan $A D$, et al. International mobile-health intervention on physical activity, sitting, and weight: the Stepathlon cardiovascular health study. J Am Coll Cardiol. 2016;67(21):2453-63. http://dx.doi.org/10.1016/j.jacc.2016.03.472

38. Schulz KF, Altman DG, Moher D. CONSORT 2010 statement: updated guidelines for reporting parallel group randomised trials. BMC Medicine. 2010;8:18. http://dx.doi.org/10.1186/1741-7015-8-18

39. Schoeppe S, Duncan MJ, Badland H, Oliver M, Curtis C. Associations of children's independent mobility and active travel with physical activity, sedentary behaviour and weight status: a systematic review. J Sci Med Sport. 2013;16(4):312-9. http://dx.doi.org/10.1016/j.jsams.2012.11.001

40. Ramachandran A, Snehalatha C, Ram J, Selvam S, Simon M, Naditha A, et al. Effectiveness of mobile phone messaging in prevention of type 2 diabetes by lifestyle modification in men in India: a prospective, parallel-group, randomised controlled trial. Lancet Diabetes Endocrinol. 2013;1(3):191-8. http://dx.doi.org/10.1016/S2213-8587(13)70067-6 .

41. Ram J, Selvam S, Snehalatha C, Naditha A, Simon M, Shetty AS, et al. Improvement in diet habits, independent of physical activity helps to reduce incident diabetes among prediabetic Asian Indian men. Diabetes Res Clin Pract. 2014;106(3):491-5. http://dx.doi.org/10.1016/j.diabres.2014.09.043 . 
42. Shetty AS, Chamukuttan S, Nanditha A, Raj RK, Ramachandran A. Reinforcement of adherence to prescription recommendations in Asian Indian diabetes patients using short message service (SMS)-a pilot study. J Assoc Physicians India. 2011;59:711-4.

43. Tamban C, Isip-Tan IT, Jimeno C. Use of short message services (SMS) for the management of type 2 diabetes mellitus: a randomized controlled trial. J ASEAN Fed Endocr Soc. 2013;28(2):143-9. http://dx.doi.org/10.15605/jafes.028.02.08 .

44. Sriramatr S, Berry TR, Spence JC. An Internet-based intervention for promoting and maintaining physical activity: a randomized controlled trial. Am J Health Behav. 2014;38(3):430-9.

45. Shahid M, Mahar SA, Shaikh S, Shaikh ZU. Mobile phone intervention to improve diabetes care in rural areas of Pakistan: a randomized controlled trial. J Coll Physicians Surg Pak. 2015;25(3):166-71. https://umw.ncbi.n/m.nih.gov/pubmed/25772954.

46. Müller AM, Khoo S, Morris T. Text messaging for exercise promotion in older adults from an upper-middle-income country: randomized controlled trial. J Med Internet Res. 2016;18(1):e5. http://dx.doi.org/10.2196/jmir.5235

47. Zolfaghari M, Mousavifar SA, Pedram S, Haghani H. The impact of nurse short message services and telephone follow-ups on diabetic adherence: which one is more effective? J Clin Nurs. 2012;21(13-14):1922-31. http://dx. doi.org/10.1111/j.1365-2702.2011.03951.x .

48. Bombem KCM, Silva Canella D, Henrique Bandoni D, Constante Jaime P. Impact of an educational intervention using e-mail on diet quality. Nutr Food Sci. 2014;44(5):431-42. http://dx.doi.org/10.1108/NFS-02-2013-0034

49. Pfammatter A, Spring B, Saligram N, Dave R, Gowda A, Blais L, et al. mHealth intervention to improve diabetes risk behaviors in India: a prospective, parallel group cohort study. J Med Internet Res. 2016;18(8):e207. http://dx. doi.org/10.2196/jmir.5712.

50. Rotheram-Borus MJ, Tomlinson M, Gwegwe M, Comulada WS, Kaufman N, Keim M. Diabetes buddies: peer support through a mobile phone buddy system. Diabetes Educ. 2012;38(3):357-65. http://dx.doi.org/10.1177/0145721712444617.

51. Chen P, Chai J, Cheng J, Li K, Xie S, Liang H, et al. A smart web aid for preventing diabetes in rural China: preliminary findings and lessons. J Med Internet Res. 2014;16(4):e98. http://dx.doi.org/10.2196/jmir.3228

52. Nurgul K, Nursan C, Dilek K, Over OT, Sevin A. Effect of web-supported health education on knowledge of health and healthy-living behaviour of female staff in a turkish university. Asian Pac J Cancer Prev. 2014;16(2):48994. http://dx.doi.org/10.7314/APJCP.2015.16.2.489.

53. Head KJ, Noar SM, lannarino NT, Grant Harrington N. Efficacy of text messaging-based interventions for health promotion: a meta-analysis. Soc Sci Med. 2013;97:41-8. http://dx.doi.org/10.1016/j.socscimed.2013.08.003

54. Fanning J, Mullen SP, McAuley E. Increasing physical activity with mobile devices: a meta-analysis. J Med Internet Res. 2012;14(6):e161. http://dx.doi. org/10.2196/jmir.2171

55. Lewis ZH, Lyons EJ, Jarvis JM, Baillargeon J. Using an electronic activity monitor system as an intervention modality: A systematic review. BMC Public Health. 2015;15:585. http://dx.doi.org/10.1186/s12889-015-1947-3

56. Gurman TA, Rubin SE, Roess AA. Effectiveness of mHealth behavior change communication interventions in developing countries: a systematic review of the literature. J Health Commun. 2012;17(1):82-104. http://dx.doi.org/10. 1080/10810730.2011.649160.

57. Déglise C, Suggs LS, Odermatt P. Short message service (SMS) applications for disease prevention in developing countries. J Med Internet Res. 2012; 14(1):e3. http://dx.doi.org/10.2196/jmir.1823

58. Bloomfield GS, Vedanthan R, Vasudevan L, Kithei A, Were M, Velazquez EJ. Mobile health for non-communicable diseases in Sub-Saharan Africa: a systematic review of the literature and strategic framework for research. Global Health. 2014;10:49. http://dx.doi.org/10.1186/1744-8603-10-49 .

59. Hall CS, Fottrell E, Wilkinson S, Byass P. Assessing the impact of mHealth interventions in low-and middle-income countries-what has been shown to work? Glob Health Action. 2014;7:256060. http://dx.doi.org/10.3402/gha.v7.25606 .

60. Peiris D, Praveen D, Johnson C, Mogulluru K. Use of mHealth systems and tools for non-communicable diseases in low- and middle-income countries: a systematic review. J Cardiovasc Transl Res. 2014;7(8):677-91. http://dx.doi. org/10.1007/s12265-014-9581-5.

61. Beratarrechea A, Lee AG, Willner JM, Jahangir E, Ciapponi A, Rubinstein A. The impact of mobile health interventions on chronic disease outcomes in developing countries: a systematic review. Telemed J E Health. 2014;20(1): 75-82. http://dx.doi.org/10.1089/tmj.2012.0328 .

62. Lyzwinski LN. A systematic review and meta-analysis of mobile devices and weight loss with an intervention content analysis. J Pers Med. 2014;4(3): 311-85. http://dx.doi.org/10.3390/jpm4030311.
63. O'Reilly GA, Spruijt-Metz D. Current mHealth technologies for physical activity assessment and promotion. Am J Prev Med. 2013;45(4):501-7. http:// dx.doi.org/10.1016/j.amepre.2013.05.012

64. Hingle $M$, Patrick $H$. There are thousands of apps for that: navigating mobile technology for nutrition education and behavior. J Nutr Educ Behav. 2016; 48(3):213-218.e1. http://dx.doi.org/10.1016/j.jneb.2015.12.009

65. Ramadas A, Chan CKY, Oldenburg B, Hussien Z, Quek KF. A web-based dietary intervention for people with type 2 diabetes: development, implementation, and evaluation. Int J Behav Med. 2015;22(3):365-73. http:// dx.doi.org/10.1007/s12529-014-9445-z .

66. Ang YK, Mirnalini K, Zalilah MS. A workplace email-linked website intervention for modifying cancer-related dietary and lifestyle risk factors: rationale, design and baseline findings. Malays J Nutr. 2013;19(1):37-51.

67. Noah SA, Abdullah SN, Shahar S, Abdul-Hamid H, Khairudin N, Yusoff M, et al. DietPal: a Web-based dietary menu-generating and management system. J Med Internet Res. 2004;6(1):e4.

68. Haddad NS, Istepanian R, Philip N, Khazaal FA, Hamdan TA, Pickles T, et al. A feasibility study of mobile phone text messaging to support education and management of type 2 diabetes in Iraq. Diabetes Technol Ther. 2014;16(7): 454-9. http://dx.doi.org/10.1089/dia.2013.0272 .

69. Hussain MI, S Naqvi B, Ahmed I, Ali N. Hypertensive patients' readiness to use of mobile phones and other information technological modes for improving their compliance to doctors' advice in Karachi. Pak J Med Sc. 2015;31(1):9-13. http://dx.doi.org/10.12669/pjms.311.5469 .

70. Esau N, Koen N, Herselman MG. Adaptation of the RenalSmart ${ }^{\oplus}$ web-based application for the dietary management of patients with diabetic nephropathy. S Afr J Clin Nutr. 2013;26(3):132-40.

71. Hopewell S, Loudon K, Clarke MJ, Oxman AD, Dickersin K. Publication bias in clinical trials due to statistical significance or direction of trial results. Cochrane Database Syst Rev. 2009;1:MR000006. http://dx.doi.org/10.1002/ 14651858.MR000006.pub3

72. Short CE, Rebar AL, Plotnikoff RC, Vandelanotte C. Designing engaging online behaviour change interventions: A proposed model of user engagement. Eur Health Psychol. 2015;17(1):32-8.

73. Yardley L, Morrison L, Bradbury K, Muller I. The person-based approach to intervention development: application to digital health-related behavior change interventions. J Med Internet Res. 2015;17(1):e30. http://dx.doi.org/ 10.2196/jmir.4055

74. Hall AK, Cole-Lewis H, Bernhardt JM. Mobile text messaging for health: a systematic review of reviews. Annu Rev Public Health. 2015;36:393-415. http://dx.doi.org/10.1146/annurev-publhealth-031914-122855

\section{Submit your next manuscript to BioMed Central and we will help you at every step:}

- We accept pre-submission inquiries

- Our selector tool helps you to find the most relevant journal

- We provide round the clock customer support

- Convenient online submission

- Thorough peer review

- Inclusion in PubMed and all major indexing services

- Maximum visibility for your research

Submit your manuscript at www.biomedcentral.com/submit
) Biomed Central 